\title{
O EFEITO DA LATERALIDADE EM TESTE DE FALA NO RUÍDO EM NORMO-OUVINTES
}

\section{The handedness effect in speech test in noise in normal hearing subjects}

\author{
Karine Thaís Becker ${ }^{(1)}$, Maristela Julio Costa ${ }^{(2)}$, Larissa Lautenschlager ${ }^{(3)}$, \\ Larissa Cristina Schuster( ${ }^{(4)}$, Tais Regina Hennig(5), Tania Maria Tochetto(5)
}

\section{RESUMO}

Objetivo: verificar o efeito da lateralidade em teste monoaural, na presença de ruído competitivo, que utiliza sentenças como estímulo em indivíduos normo-ouvintes. Método: 81 indivíduos normo-ouvintes, com idades entre 18 e 44 anos, divididos em 2 grupos de acordo com a lateralidade - 45 indivíduos destros e 36 canhotos -, foram avaliados por meio do teste Listas de Sentenças em Português (LSP), e obtida a relação sinal-ruído (S/R) para cada orelha. Resultados: os valores médios obtidos para as relações $S / R$ na orelha direita, para os indivíduos destros e canhotos, foram, respectivamente, $-6,5 \mathrm{~dB}$ e $-4,5 \mathrm{~dB}$. Para a orelha esquerda, foram $-7,0 \mathrm{~dB}$ e $-7,5 \mathrm{~dB}$. As orelhas direitas apresentaram resultados significantemente piores, tanto nos indivíduos destros, como nos canhotos. No entanto a orelha direita dos canhotos foi sensivelmente pior em comparação a dos destros. Conclusão: existe diferença quanto ao reconhecimento de fala, na presença de ruído competitivo, em indivíduos destros e canhotos. A orelha direita apresentou pior desempenho em ambos os grupos, sendo que esse resultado negativo é mais acentuado nos canhotos.

DESCRITORES: Audição; Fala; Percepção da Fala; Lateralidade Funcional; Dominância Cerebral

\section{INTRODUÇÃO}

$\mathrm{Na}$ pesquisa sobre o processamento da linguagem, a lateralização da função pode ser

(1) Fonoaudióloga do Projeto Promoção da Saúde Auditiva da Universidade Federal de Santa Maria; Mestranda em Distúrbios da Comunicação Humana pela Universidade Federal de Santa Maria.

(2) Fonoaudióloga; Professora Adjunta do Departamento de Fonoaudiologia da Universidade Federal de Santa Maria; Doutora em Ciências dos Distúrbios da Comunicação Humana pela Universidade Federal de São Paulo.

(3) Fonoaudióloga, Mestre em Distúrbios da Comunicação Humana da Universidade Federal de Santa Maria - UFSM.

(4) Fonoaudióloga do Projeto Promoção da Saúde Auditiva da Universidade Federal de Santa Maria; Mestranda em Distúrbios da Comunicação Humana pela Universidade Federal de Santa Maria.

(5) Fonoaudióloga do Projeto Promoção da Saúde Auditiva da Universidade Federal de Santa Maria; Mestranda em Distúrbios da Comunicação Humana pela Universidade Federal de Santa Maria.

(6) Fonoaudióloga, Doutora em Ciências dos Distúrbios da Comunicação Humana - Campo Fonoaudiológico pela UNIFESP, Professora Associada do Departamento de Fonoaudiologia da UFSM.

Conflito de interesses: inexistente usada para explicar diferenças sutis na habilidade cognitiva ${ }^{1}$.

O processamento mental da fala é uma das funções de maior assimetria cortical, na qual o hemisfério esquerdo tem maior competência para tal função em associação com o hemisfério direito. Isso retira o aspecto robótico e favorece o colorido da comunicação interpessoal através da prosódia ${ }^{2,3}$.

Várias pesquisas têm revelado diferença cerebral, estrutural ou funcional, entre destros e canhotos. Essas diferenças têm sido descritas na lateralização anatômica de várias estruturas, na morfologia do corpo caloso ou na eficiência da interação hemisférica ${ }^{4,5}$.

Estudos apontaram estratégias corticais diferentes para a especialização hemisférica entre destros e canhotos, sendo que $15 \%$ da população de sinistros podem utilizar o hemisfério direito ou ambos para essa função ${ }^{2,6}$.

Determinar clinicamente esta assimetria pode não ser tarefa fácil, pois diferentes avaliações podem vir a ter conclusões diferentes ${ }^{3,7}$. Encontrase, na literatura, uma série de estudos que utilizam diferentes formas de avaliação (monoaural, binaural 
e dicótica). Porém nem sempre a assimetria entre as orelhas foi observada ${ }^{8-11}$.

Alguns autores relataram que a apresentação monoaural pode falhar para fornecer provas convincentes das diferenças hemisféricas ${ }^{12}$. Por outro lado, a apresentação binaural dos sons da fala muitas vezes não revela assimetrias hemisféricas significantes na atividade neural ${ }^{13}$, bem como estudos com uso da apresentação dicótica da fala não resultaram consistentemente em assimetrias do hemisfério esquerdo ${ }^{14}$.

O tipo de estímulo empregado também aponta diferenças na avaliação da assimetria. Estudos mostraram que as áreas temporais cerebrais são ativadas diferentemente ao serem utilizados estímulos de fala distintos - sílabas, palavras e sentenças ${ }^{12,13}$. Em um destes estudos ${ }^{13}$, a lateralização não foi observada com o uso de sílabas, mas ocorreu ao nível da palavra.

No Brasil, o estudo pioneiro para a avaliação do reconhecimento de fala utilizando sentenças como estímulo ${ }^{15}$ resultou em um teste composto por Listas de Sentenças em Português (LSP) com a finalidade de fornecer informações sobre a real habilidade de reconhecimento da mensagem falada em situações mais próximas as da vida diária. Este teste apresenta-se gravado em CD e contém oito listas de sentenças em português, foneticamente balanceadas, e um ruído com espectro de fala, gravados em canais independentes. Isso permite a apresentação das sentenças tanto no silêncio, quanto no ruído, com intensidades de apresentação diferentes ${ }^{16}$.

Sendo assim, o objetivo do presente estudo foi verificar o efeito da lateralidade em teste monoaural, na presença de ruído competitivo, que utiliza sentenças como estímulo em indivíduos normo-ouvintes.

\section{MÉTODO}

Este estudo foi realizado no Laboratório de Próteses Auditivas do Serviço de Atendimento Fonoaudiológico da Universidade Federal de Santa Maria, no período de fevereiro a junho de 2010, a partir do Projeto "Pesquisa e base de dados em saúde auditiva", registrado no GAP do Centro de Ciências da Saúde sob o no 019731.

\section{Sujeitos}

Participaram do estudo 81 sujeitos adultos, com idades entre 18 e 44 anos, divididos em dois grupos: Grupo 1 - indivíduos destros - e Grupo 2 - indivíduos canhotos. Essa divisão se deu través da aplicação da versão resumida do Inventário de Edinburgh ${ }^{17}$.
O grupo 1 ficou composto por 45 sujeitos, sendo 24 do sexo masculino e 21 do sexo feminino. O grupo 2 ficou constituído por 36 sujeitos, 18 do sexo masculino e 18 do sexo feminino.

Todos os sujeitos receberam orientações sobre os objetivos, justificativa e metodologia do estudo proposto e assinaram o Termo de Consentimento Livre e Esclarecido.

Os participantes eram alunos de graduação ou pós-graduação, socialmente ativos e produtivos.

\section{Critérios}

Foram utilizados como critérios de inclusão: limiares de audibilidade inferiores a $25 \mathrm{~dB}$ NA nas frequências de 500 a $4000 \mathrm{~Hz}^{18}$ e ausência de queixas de zumbido e hiperacusia.

\section{Procedimentos}

- anamnese: questionário para a obtenção de dados pessoais, nível de escolaridade, queixas auditivas e história otológica;

- inspeção visual do meato acústico externo;

- obtenção dos limiares auditivos nas freqüências de 500 a 4000 Hz;

- pesquisa do limiar de reconhecimento de fala LRF;

- pesquisa dos limiares de reconhecimento de sentenças no ruído (LRSR). Estes foram obtidos por meio da aplicação do teste Listas de Sentenças em Português (LSP) ${ }^{16}$.

Esse material apresenta-se gravado em CD e contém oito listas de sentenças e um ruído com espectro de fala, gravados em canais independentes, permitindo a apresentação das sentenças no ruído, com intensidades de apresentação diferentes.

Antes de iniciar o teste com cada indivíduo, a saída de cada canal do $C D$ foi calibrada pelo VU-meter do audiômetro. $\mathrm{O}$ tom de $1 \mathrm{kHz}$ presente no mesmo canal do $C D$ em que estão gravadas as sentenças, bem como o ruído mascarante presente no outro canal, foram colocados no nível zero.

As listas de sentenças e o ruído competitivo foram apresentados de forma monoaural e ipsilateralmente, por meio de fones auriculares, permitindo a avaliação das orelhas separadamente. As listas de sentenças utilizadas estão descritas na Figura 1. Foram utilizadas diferentes listas de sentenças, uma para cada condição de teste, a fim de eliminar a possibilidade de melhor desempenho devido à memorização das sentenças. $O$ uso de diferentes listas não foi considerado uma variável, pois as listas aplicadas são equivalentes entre si ${ }^{19}$. 


\section{LISTA 1A}

1. Não posso perder o ônibus.

2. Vamos tomar um cafezinho.

3. Preciso ir ao médico.

4. A porta da frente está aberta.

5. A comida tinha muito sal.

6. Cheguei atrasado para a reunião.

7. Vamos conversar lá na sala.

8. Depois liga pra mim.

9. Esqueci de pagar a conta.

10. Os preços subiram ontem.

11. O jantar está na mesa.

12. As crianças estão brincando.

13. Choveu muito neste fim-de-semana.

14. Estou morrendo de saudade.

15. Olhe bem ao atravessar a rua.

16. Preciso pensar com calma.

17. Guardei o livro na primeira gaveta.

18. Hoje é meu dia de sorte.

19. O sol está muito quente.

20. Sua mãe acabou de sair de carro.

\section{LISTA 3B}

1. Ela acabou de bater o carro.

2. É perigoso andar nessa rua.

3. Não posso dizer nada.

4. A chuva foi muito forte.

5. Os preços subiram na segunda.

6. Esqueci de levar a bolsa.

7. Os pães estavam quentes.

8. Elas já alugaram uma casa na praia.

9. Meu irmão viajou de manhã.

10. Não encontrei meu filho.

\section{LISTA 4B}

1. Sua mãe pôs o carro na garagem.

2. O aluno quer assistir ao filme.

3. Ainda não pensei no que fazer.

4. Essa estrada é perigosa.

5. Não paguei a conta do bar.

6. Meu filho está ouvindo música.

7. A chuva inundou a rua.

8. Amanhã não posso almoçar.

9. Ela viaja em dezembro.

10. Você teve muita sorte.

Figura 1 - Listas de Sentenças em Português (LSP)

As sentenças foram aplicadas na seguinte ordem:

a. Apresentação das sentenças de 1 a 10 da lista $1 \mathrm{~A}$, com a presença de ruído competitivo ipsilateralmente, na orelha direita, para familiarização do indivíduo com o teste;

b. Apresentação das sentenças de 11 a 20 da lista $1 \mathrm{~A}$, com a presença de ruído competitivo ipsilateralmente, na orelha esquerda, para familiarização do indivíduo com o teste;

c. Apresentação da lista 3B com a presença de ruído competitivo ipsilateralmente, na orelha direita.

d. Apresentação da lista 4B com a presença de ruído competitivo ipsilateralmente, na orelha esquerda.

A intensidade inicial de apresentação da primeira sentença de cada lista - intensidade necessária para cada sujeito tivesse êxito na primeira sentença de cada lista do teste - foi baseada nos resultados encontrados no treinamento acima descrito, sendo que a intensidade do ruído foi mantida constante a $65 \mathrm{~dB} N \mathrm{NA}$

A estratégia utilizada para pesquisar o LRSR foi a seqüencial ou adaptativa, ou ainda ascendentedescendente ${ }^{20}$. Esta permite mensurar o nível necessário para o indivíduo identificar, de forma correta, aproximadamente $50 \%$ dos estímulos de fala apresentados em uma determinada relação S/R.

Seguindo essa estratégia, quando o indivíduo foi capaz de reconhecer corretamente o estímulo de fala apresentado, a intensidade do mesmo foi diminuída; caso contrário, sua intensidade foi aumentada. Uma resposta só foi considerada correta quando o indivíduo repetiu, sem nenhum erro ou omissão, toda a sentença apresentada.

São sugeridos intervalos de $4 \mathrm{~dB}$ até a primeira mudança no tipo de resposta e, posteriormente, intervalos de apresentação dos estímulos de $2 \mathrm{~dB}$ entre si até o final da lista ${ }^{20}$. Porém, devido às possibilidades técnicas do equipamento disponível para a realização desta pesquisa, foram utilizados intervalos de apresentação das sentenças de $5 \mathrm{~dB}$ e 2,5 $\mathrm{dB}$, respectivamente.

É importante salientar ainda que, no primeiro estudo utilizando o LSP realizado com fones auriculares $^{21}$, foi observado uma diferença de $7 \mathrm{~dB}$ entre o volume de gravação dos dois sinais apresentados (fala e ruído), sendo que as sentenças estão gravadas em uma intensidade média de 7 $\mathrm{dB}$ abaixo da intensidade do ruído. Por esta razão, a autora do teste referiu que, nas avaliações realizadas com fones auriculares, é necessário que seja 
subtraído $7 \mathrm{~dB}$ dos valores médios de fala apresentados e observados no dial do equipamento, procedimento este adotado nesta pesquisa.

Os níveis de apresentação das sentenças foram anotados para posterior cálculo da média a partir dos valores onde houve mudança no tipo de resposta e, então, subtraídos os $7 \mathrm{~dB}$ acima mencionados, resultaram no LRSR.

Por último foi calculada a relação sinal/ruído (S/R) subtraindo-se o valor do LRSR do nível de intensidade do ruído (65 dB NA)

A variável considerada no estudo foi o LRSR, expresso por meio da relação $S / R$.

\section{Materiais}

Para a obtenção das medidas, foram utilizados: um audiômetro digital de dois canais, marca Fonix, modelo FA-12, tipo I e fones auriculares tipo TDH-39P, marca Telephonics. As sentenças e o ruído foram apresentados utilizando-se um Compact Disc Player Digital Toshiba - 4149 acoplado ao audiômetro descrito.

Este estudo foi aprovado pela Comissão de Ética em Pesquisa (CEP) da UFSM com certificado de $n^{\circ}$ 0138.0.243.000.06.

\section{Análise dos dados}

Foi realizada a análise descritiva dos valores e, em seguida, os dados coletados foram submetidos a tratamento estatístico, primeiramente por meio da análise do comportamento das variáveis. Após constatar que a distribuição dos dados é normal, foi aplicado o teste t Pareado. Este teste tem como finalidade verificar se a diferença entre as médias das relações $S / R$ da orelha direita e da orelha esquerda dos sujeitos de cada grupo teve significância ou não. Foi considerado nível de significância estatística de $p \leq 0,05$ (5\%).

\section{RESULTADOS}

A seguir, estão apresentados os resultados obtidos com as avaliações realizadas nos 81 indivíduos, sendo 45 destros (Grupo 1) e 36 canhotos (Grupo 2).

As Tabelas 1 e 2 apresentam os dados obtidos a partir da análise comparativa dos resultados médios da relação $S / R$, por orelha, dos grupos estudados.

Nas Tabelas 3 e 4 estão expostos os resultados da análise comparativa das orelhas direitas e das orelhas esquerdas entre os grupos.

Tabela 1 - Média, desvio padrão (dp), valores mínimos, máximos da relação s/r do grupo 1 para as orelhas direitas e esquerdas

\begin{tabular}{cccccc}
\hline Destros $\mathbf{n = 4 5}$ & Média & DP & Mín. & Máx. & Valor de $\mathbf{p}$ \\
\hline OD & $-6,5$ & 2,29 & $-12,8$ & -2 & \multirow{2}{*}{$0,0466^{*}$} \\
OE & $-7,0$ & 2,04 & $-11,7$ & $-2,5$ & \\
\hline
\end{tabular}

*significância $(p \leq 0,05)$

Teste t Pareado

Tabela 2 - Média, desvio padrão (dp), valores mínimos, máximos da relação s/r do grupo 2 para as orelhas direitas e esquerdas

\begin{tabular}{cccccc}
\hline $\begin{array}{c}\text { Canhotos } \\
\mathbf{n = 3 6}\end{array}$ & Média & DP & Mín. & Máx. & Valor de $\mathbf{p}$ \\
\hline OD & $-4,5$ & 2,25 & $-9,1$ & $-0,4$ & $0,0001^{*}$ \\
OE & $-7,5$ & 2,13 & $-13,2$ & -2 & 0 \\
\hline
\end{tabular}

*significância $(p \leq 0,05)$

Teste t Pareado

Tabela 3 - análise comparativa das médias das orelhas direitas entre os grupos 1 e 2

\begin{tabular}{ccc}
\hline $\mathbf{N}=\mathbf{8 1}$ & Media & Valore de $\mathbf{p}$ \\
\hline Destros & $-6,45$ & $0,0001^{*}$ \\
Canhotos & $-4,52$ & \\
\hline
\end{tabular}

*significância $(p \leq 0,05)$

Teste t Pareado
Tabela 4 - Análise comparativa das médias das orelhas esquerdas entre os grupos 1 e 2

\begin{tabular}{ccc}
\hline $\mathbf{N}=\mathbf{8 1}$ & Media & Valore de $\mathbf{p}$ \\
\hline 1- Destros & -7.05 & 0,1583 \\
2- Canhotos & $-7,52$ & \\
\hline
\end{tabular}

significância $(p \leq 0,05)$ - não houve

Teste t Pareado 


\section{DISCUSSÃO}

O efeito da lateralidade em teste monoaural, na presença de ruído competitivo, que utiliza sentenças como estímulo, foi estudado em indivíduos destros e canhotos no presente estudo.

Os sujeitos do grupo 1 (destros), obtiveram valores médios de relação $S / R$ para a OD de $-6,5$ $\mathrm{dB}$ e para a OE de $-7,0 \mathrm{~dB}$. Os sujeitos do grupo 2 (canhotos) obtiveram médias para a OD de $-4,5 \mathrm{~dB}$ e para a OE de $-7,5 \mathrm{~dB}$.

Antes de iniciar a discussão dos achados, vale ressaltar que quanto maior o valor negativo da relação $S / R$, mais desfavorável ela será e melhor o desempenho dos sujeitos frente ao ruído competitivo.

A análise comparativa dos resultados obtidos entre as orelhas direitas e esquerdas dos sujeitos de cada grupo constatou diferença estatisticamente significante entre os valores médios obtidos para as relações $S / R$ em ambos os grupos, indicando que as orelhas direitas apresentaram resultados significantemente piores, tanto nos indivíduos destros, como nos canhotos. Assim os sujeitos necessitaram de relações $S / R$ mais favoráveis nas ODs e mais desfavoráveis nas OEs, para reconhecer, em média, $50 \%$ dos estímulos de fala apresentados diante de ruído competitivo.

Na comparação do desempenho dos indivíduos entre os grupos, as médias das ODs chamaram atenção. Os destros obtiveram média de $-6,5 \mathrm{~dB}$ e os canhotos média de $-4,5 \mathrm{~dB}$. A análise estatística revelou diferença significante entre esses valores, com pior desempenho dos canhotos. $\mathrm{Na}$ comparação dos valores da relação S/R da OE, não foi revelada diferença significante. Dessa forma, destros e canhotos obtiveram desempenho semeIhante com a OE, mas diferiram quanto ao desempenho na OD.

$\mathrm{Na}$ literatura, comumente, são encontrados relatos que argumentam sobre o processamento da linguagem e a lateralização para o hemisfério esquerdo (HE), o que resulta em uma melhor percepção dos estímulos verbais na orelha direita em relação à esquerda ${ }^{1,6,12,22-24}$.

Como no presente estudo foi verificado pior desempenho na OD em ambos os grupos estudados, estes resultados não corroboram com os achados da literatura que verificaram a vantagem da orelha.

Entretanto, segundo um estudo recente, a função da linguagem pode ocorrer no hemisfério direito em áreas homólogas às áreas de Broca e de Wernicke em indivíduos destros ${ }^{1}$, refletindo em melhora no desempenho da orelha esquerda. Quando a vantagem ocorre na OE, maior especialização do hemisfério direito (HD) pode ser observada ${ }^{25}$.

Alguns autores inferem que os efeitos de lateralidade podem ser influenciados pelo tipo de estímulo, tarefas e variações individuais, assim como pela complexidade e pelas exigências da tarefa ${ }^{3,13,25}$.

Sabe-se que repetir sentenças na presença de ruído competitivo requer atividade auditiva mais complexa para que o estímulo de fala seja processado do que repetir sílabas ou palavras. Estudos empregando sentenças como estímulo investigaram a influência do hemisfério direito (áreas homólogas à área de Broca e à área de Wernicke) na função da linguagem usando imagens de ressonância magnética ${ }^{26}$. Estes autores encontraram aumento na ativação dessas áreas quando as demandas cognitivas foram aumentadas. Da mesma forma, foi evidenciada maior ativação no lobo parietal direito como resultado do aumento da dificuldade da tarefa ${ }^{27}$.

Dessa forma, evidencia-se que o aumento do envolvimento do hemisfério direito pode resultar em aumento do desempenho em várias tarefas de linguagem ${ }^{1}$. Assim os achados aqui demonstrados concordam com estes estudos, em que a maior ativação do hemisfério direito pode resultar em melhor desempenho da OE em indivíduos destros.

Porém, mesmo com tais aferições, ainda permanece aberta a questão referente ao pior desempenho demonstrado pelos indivíduos canhotos com a OD em relação aos destros.

Vários estudos têm revelado diferença cerebral, estrutural ou funcional entre destros e canhotos ${ }^{4}$. Quando estes indivíduos são comparados, alguns estudos não encontraram diferenças quanto à lateralidade auditiva, enquanto outros encontraram redução da vantagem da OD em canhotos e outros, ainda, encontraram assimetria invertida, com vantagem da orelha esquerda em destros ${ }^{28,29}$.

Em resposta às tarefas contidas em inventários de preferências manuais, em relação aos destros, os canhotos quase sempre exibem um alto nível de variabilidade ${ }^{30}$.

Como já mencionado anteriormente, a avaliação da lateralidade pode sofrer influência de alguns fatores, como experiência, procedimento, instruções da tarefa, atenção ${ }^{3}$. Assim, quando se avalia a lateralidade, a medida obtida reflete uma combinação de componentes estruturais e de atenção. Estes efeitos de atenção são determinados pelo contexto dado à pessoa na situação experimental e pelos próprios processos do pensamento. Instruções que permitam aos participantes o controle de atenção são susceptíveis a produzir melhor desempenho para um lado ou para o outro ${ }^{31}$. 
Durante a realização da anamnese, vários indivíduos referiram perceber melhor performance com a OE, sendo esta a orelha preferida para atender o telefone e ouvir música por meio de fones de ouvido, por exemplo. Portanto, uma possível hipótese para os resultados encontrados seria a maior atenção prestada pelos indivíduos quando a OE foi avaliada, somada a possíveis fatores estruturais e funcionais diferentes aos dos destros, conforme relatado por muitos estudiosos. Como conseqüência o desempenho da OD seria pior.

Estudos demonstraram que, em indivíduos destros a atenção dirigida (ou forçada) para a direita, geralmente aumenta a vantagem da OD e a atenção dirigida para a esquerda em geral reduz ou reverte esta vantagem ${ }^{6}$. O mesmo fato pode ter ocorrido com os canhotos neste estudo.

Por causa da inversão da vantagem da orelha, tanto em indivíduos destros quanto canhotos, alguns autores mencionaram que um dos principais fatores que interferem na vantagem da orelha é a atenção ${ }^{6}$.

Porém demais fatores intrínsecos relacionados ao processamento do som através das vias e redes neuronais possivelmente, estão envolvidos neste processo, o que justificaria o desempenho inferior da OD.

Desta forma pode-se inferir que foi verificado efeito da lateralidade no reconhecimento de sentenças no ruído nos grupos estudados, com pior desempenho, estatisticamente significante, nas orelhas direitas. A habilidade de reconhecer a fala na presença de ruído competitivo não depende apenas dos limiares audiométricos, mas, sim, de um conjunto de fatores individuais que determinam como cada pessoa é capaz de processar a informação recebida ${ }^{32}$.

Assim sendo, com base na literatura e nos resultados aqui encontrados, verifica-se que a lateralidade influencia as habilidades de processamento auditivo, neste caso aqui investigado principalmente as habilidades de figura-fundo e atenção seletiva ${ }^{33,34}$ e, portanto, torna-se interessante a realização de estudos mais aprofundados que incluam outros instrumentos de avaliação, inclusive utilizando técnicas de neuroimagem, a fim de entender melhor todos os processos envolvidos nesta tarefa tão complexa e fascinante que é o processamento auditivo.

\section{CONCLUSÕES}

- Foi verificada diferença quanto ao reconhecimento de sentenças, na presença de ruído competitivo, em indivíduos destros e canhotos;

- Indivíduos destros e canhotos obtiveram desempenho semelhante com a orelha esquerda, mas diferiram quanto ao desempenho na orelha direita;

- A orelha direita foi significantemente pior nos canhotos em comparação aos destros.

\begin{abstract}
Purpose: to check the handedness effects in monaural test using sentences as stimulus with background noise in adults with normal hearing. Method: 81 subjects, with age between 18 and 44 , divided in two groups according to handedness - 45 right-handers and 36 left-handers, were evaluated through the Portuguese Sentences Lists test (PSL) then we obtained the signal to noise ratio for each ear. Results: the mean values obtained as for the signal to noise ratio on right ear for the right- and left -handed subjects were respectively $-6.5 \mathrm{~dB}$ and $-4.5 \mathrm{~dB}$. On the left ear the computed results were $-7.0 \mathrm{~dB}$ and $-7.5 \mathrm{~dB}$. The right ears showed significantly poor results in both right-handed subjects, as well as in left-handers. As left-handed subjects right ears was significantly poor compared to that of right-handers. Conclusion: there is difference in speech recognition with background noise in right- and left -handed subjects. The right ears showed poor performance in both groups, and this worsening is more emphasized in the left-handers.
\end{abstract}

KEYWORDS: Hearing; Speech; Speech Perception; Functional Laterality; Dominance, Cerebral 


\section{REFERÊNCIAS}

1. $H M$ van Ettinger-Veenstra, Ragnehed $M$, Hällgren M, Karlsson T, Landtblom AM, Lundberg $P$ et al. Right-hemispheric brain activation correlates to language performance. Neurolmage. 2010; 49:3481-8.

2. Kent RD. Research on speech motor control and its disorders: A review and prospective. Ms. Waisman Center, University of Wisconsin-Madison, Wisconsin, 2000.

3. Fischer J, Teufel C, Drolet M, Patzelt A, Rübsamen $\mathrm{R}$, Yvesvon Cramon $\mathrm{D}$ et al. Orienting asymmetries and lateralized processing of sounds in humans. BMC Neuroscience. 2009; 10 (supl. 1)14. doi:10.1186/1471-2202-10-14.

4. Cherbuin N, Brinkman C. Hemispheric interactions are different in left-handed individuals. Neuropsychology. 2006;20:700-7.

5. Klöppel S, Vongerichten A, Eimeren $T$, Frackowiak RSJ, Siebner HR. Can Left-Handedness be Switched? Insights froman Early Switch of Handwriting. The Journal of Neuroscience. 2007; 27(29):7847-53.

6. Foundas AL, Corey DM, Hurley MM, Heilman $\mathrm{KM}$. Verbal dichotic listening in right and left-handed adults: Laterality effects of directed attention. Cortex. 2006;42: 79-86.

7. Stroobant N, Buijs D, Vingerhoets G. Variation in brain lateralization during various language tasks: $A$ functional Transcranial Doppler study. Behavioural Brain Research. 2009; 199: 190-6.

8. Samelli AG, Schochat E. Estudo da vantagem da orelha direita em teste de detecção de gap. Rev. Bras. Otorrinolaringol. 2008; 74(2): 235-40.

9. Sætrevik B, Hugdahl K. Priming inhibits the right ear advantage in dichotic listening: Implications for auditory laterality. Neuropsychologia. 2007; 45: 282-7.

10. Oliveira CT, Silva VF, Silva JRV. Mapeamento da atividade cortical da área de broca durante uma tarefa de processamento mental da fala em indivíduos mono-hemisfericitos direito. Fitness \& Performance Journal. 2006; 5(4): 236-42.

11. Behne N, Wendt B, Scheich H, Brechmann A. Contralateral white noise selectively changes left human auditory cortex activity in a lexical decision task. Journal of Neurophysiology. 2006; 95(4): 2630-7.

12. Stefanatos GA, Joe WQ, Aguirre GK, Detre JA, Wetmore G. Activation of human auditory cortex during speech perception: Effects of monaural, binaural, and dichotic presentation. Neuropsychologia. 2008; 46: 301-15.

13. Poeppel D. Auditory lexical decision, categorical perception, and FM direction discrimination differentially engage left and right auditory cortex. Neuropsychologia. 2004; 42: 183-200.

14. Hugdahl K. The effect of stimulus intensity on the right ear advantage in dichotic listening. Neuroscience Letters. 2008; 431:90-4.

15. Costa MJ. Desenvolvimento de listas de sentenças em português [Tese]. São Paulo (SP): Universidade Federal de São Paulo/ Escola Paulista de Medicina; 1997.

16. Costa MJ. Listas de sentenças em português: apresentação e estratégias de aplicação na audiologia. Santa Maria : Pallotti; 1998.

17. Oldfield RC. The assessment and analysis of handedness: The Edinburgh Handedness Inventory. Neuropsychologoia. 1971; 9:97-118.

18. Davis H, Silverman SR. Interpretação dos resultados da avaliação audiológica. In: Santos TMM, Russo ICP. Prática da audiologia clínica. 6. ed. São Paulo: Cortez; 2007. p.291-310.

19. Santos SN, Daniel RC, Costa JC. Estudo da equivalência entre as listas de sentenças em português. Rev. CEFAC. 2009; 11(4): 673-80.

20. Levitt $H$, Rabiner LR. Use of a sequencial strategy in intelligibility testing. J Acoust Soc Am. 1967; 42:609-12.

21. Cóser PL, Costa MJ, Cóser MJS, Fukuda Y. Reconhecimento de sentenças no silêncio e no ruído em indivíduos portadores de perda induzida pelo ruído. Rev Bras de Otorrinolaringol. 2000; 66:(4):362-70.

22. Marzoli D, Tommasi L. Side biases in humans (Homo sapiens): three ecological studies on hemispheric asymmetries. Naturwissenschaften. 2009; 96:1099-106.

23. Benavides M, Peñaloza-López YR, SanchaJiménez S, Pedroza FG, Gudiño PK. Auditory and corporal laterality, logoaudiometry and monaural hearing aid gain. Acta Otorrinolaringol Esp. 2007; 58(10):458-63.

24. Iliadou V, Kaprinis S, Kandylis D, Kaprinis G. Hemispheric laterality assessment with dichotic digits testing in dyslexia and auditory processing disorder. Internarional Journal of Audiology. 2010; 49:247-52.

25. Grimshawa GM, Kwasny KM, Covell E, Johnson RA. The dynamic nature of language lateralization: effects of lexical and prosodic factors. Neuropsychologia. 2003; 41:1008-19.

26. Just MA, Carpenter PA, Timothy AK, William FE, Keith RT. Brain Activation Modulated by Sentence Comprehension. Science. 1996; 274: 114-6.

27. Dräger $B$, Jansen A, Bruchmann S, Förster AF, Pleger B, Zwitserlood P, Knecht S. How does the brain accommodate to increased task difficulty in Word finding? Afunctional MRI study. Neuro Image. 2004; 23:1152-60. 
28. Bedoin N., Ferragne E., Marsico E. Hemispheric asymmetries depend on the phonetic feature: $A$ dichotic study of place of articulation and voicing in French stops. Brain \& Language. No prelo 2010. 29. Fels G, Bruhns P, Rau S, Wiesmann M, Ilmberger J, Kegel $G$ et al. Sensitivity and reliability of language laterality assessment with a free reversed association task - a fMRI study. Eur Radiol. 2010; 20:683-95.

30. Savel S. Individual differences and left/right asymmetries in auditory space perception. I. Localization of low-frequency sounds in free field. Hearing Research. 2009; 255: 142-54.
31. Voyer D. Reliability and Magnitude of Perceptual Asymmetries in a Dichotic Word Recognition Task. Neuropsychology. 2003; 17(3): 393-401.

32. Costa MJ, lório MCM, Mangabeira Albernaz PL. Reconhecimento de fala: desenvolvimento de uma lista de sentenças em português. Acta Awho 1997; 16(4):164-73.

33. Garcia VL, Pereira LD, Fukuda Y. Atenção seletiva:PSl em crianças com distúrbio de aprendizagem. Rev Bras Otorrinolaringol. 2007;73(3):404-11.

34. Pereira LD. Sistema auditivo e desenvolvimento das habilidades auditivas. In: Ferreita LP. Tratado de Fonoaudiologia - 2ª edição. Brasil: Roca; 2009. p. 3-8.

Endereço para correspondência:

Karine Thaís Becker

Endereço: Rua Pedro Santini, 177/109-C, Nossa Senhora de Lourdes

Santa Maria - RS

CEP: 97060-480

E-mail: katthais@ @otmail.com 